\title{
BMJ Open Is there a sex difference in mortality rates for deaths occurring in paediatric intensive care units? Systematic literature review protocol
}

\author{
Ofran Almossawi (D) , ${ }^{1}$ Amanda Friend, ${ }^{2}$ Luigi Palla, ${ }^{3,4,5}$ Richard Feltbower, ${ }^{6}$ \\ Bianca De Stavola ${ }^{1}$
}

To cite: Almossawi 0 , Friend $A$, Palla L, et al. Is there a sex difference in mortality rates for deaths occurring in paediatric intensive care units? Systematic literature review protocol. BMJ Open 2021;11:e046794. doi:10.1136/ bmjopen-2020-046794

- Prepublication history and additional material for this paper is available online. To view these files, please visit the journal online (http://dx.doi.org/10. 1136/bmjopen-2020-046794).

Received 10 November 2020 Revised 07 January 2021 Accepted 21 January 2021

Check for updates

(C) Author(s) (or their employer(s)) 2021. Re-use permitted under CC BY-NC. No commercial re-use. See rights and permissions. Published by BMJ.

For numbered affiliations see end of article.

Correspondence to Mrs Ofran Almossawi; o.almossawi@ucl.ac.uk

\section{ABSTRACT}

Introduction In the general population, female children have been reported to have a survival advantage. For children admitted to paediatric intensive care units (PICUs), mortality has been reported to be lower in males despite the higher admission rates for males into intensive care. This apparent sex reversal in PICU mortality is not well studied. To address this, we propose to conduct a systematic literature review to summarise the available evidence. Our review will study the reported differences in mortality between males and females aged $0-17$, who died in a PICU, to examine if there is a difference between the two sexes in PICU mortality, and if so, to describe the magnitude and direction of this difference.

Methods and analysis Studies that directly or indirectly addressed the association between sex and mortality in children admitted to intensive care will be eligible for inclusion. Studies that directly address the association will be eligible for data extraction. The search strings were based on terms related to the population (children in intensive care), the exposure (sex) and the outcome (mortality). We used the databases MEDLINE (1946-2020), Embase (1980-2020) and Web of Science (1985-2020) as these cover relevant clinical publications. We will assess the reliability of included studies using the risk of bias in observational studies of exposures tool. We will consider a pooled effect if we have at least three studies with similar periods of follow up and adjustment variables.

Ethics and dissemination Ethical approval is not required for this review as it will synthesise data from existing studies. This manuscript is a part of a larger data linkage study, for which Ethical approval was granted. Dissemination will be via peer-reviewed journals and via public and patient groups.

PROSPERO registration number CRD42020203009.

\section{BACKGROUND}

Child mortality is a global measure of a nation's health and a top priority for the UK health system. ${ }^{1}$ Differences in mortality rates between the sexes are well documented in almost all developed countries to be in favour of higher female over male survival. ${ }^{2}$ Overall childhood mortality is very low in the UK,
Strengths and limitations of this study

- The strengths of our review include systematically evaluating all published articles that study the relationship between sex and paediatric intensive care unit (PICU) mortality where sex was both the primary exposure, or used as an adjustment variable.

- We have ensured wide coverage of the literature by selecting a broad date range.

- Although we focus on the relationship between sex and PICU mortality as a primary aim, our review is not designed to estimate causal associations.

- Limiting articles to those published in the English language will introduce bias, but the proportion of excluded articles due to publication language will be assessed and reviewed for future inclusion if $20 \%$ or more are excluded.

and in other developed countries. Office of National Statistics figures over the last two decades show downward mortality trends for both males and females, and levelling off over the last decade. A major contributor to the UK number of childhood deaths are those associated with an admission to paediatric intensive care unit (PICU), with males accounting for around $60 \%$ of total admissions. ${ }^{3}$ PICU deaths account for about $15 \%$ of all UK childhood fatalities ${ }^{3}$ and provide a sizeable population to study sex differences in childhood deaths. This led to the design and implementation of a longitudinal study of all infants admitted to UK PICUs over 11 years. The study showed higher mortality for female over male infants. ${ }^{4}$ This difference is in the opposite direction to that observed for the overall population. This difference could be due to variations in severity of disease on admission; however, accounting for currently available severity scores is not sufficient to fully explain the observed sex differences in mortality. There is a number of published 
studies that have examined this issue, reaching similar conclusions, ${ }^{5-7}$ but there is no systematic review to collate and evaluate all the available evidence on this.

For this reason, we aim to conduct a systematic literature review to study the differences in mortality rates between males and females aged $0-17$, who die in PICU. This literature review will address the questions of: whether there is a difference between the two sexes in PICU mortality, and if so, the magnitude and direction of this difference. This can be pertinent to clinical care in identifying a risk group (in this case male or female children) in which clinical practice maybe be tailored to address specific needs and allocate resources. This review is also part of a wider project using linked PICU and Hospital Episode Statistics data which aims to study differences in sex mortality and long term outcomes in England. ${ }^{8}$

\section{Aims and objectives}

Using published data, our overall primary aim is to gather evidence on whether there is a mortality difference between males and females admitted to PICU. Our secondary aim is to quantify the rates of admission to PICU for males and females. Our specific objectives are to report on the evidence with regard to:

- The rates of admission to PICU for all children aged $0-17$ years by sex.

- The rates of mortality by sex and their difference (absolute or relative, as available) in children admitted to PICU aged $0-17$ years.

- These rates overall and by any primary diagnostic groups.

\section{Review question}

Following the conclusions of the 11 year study, ${ }^{4}$ this review will focus on death while in PICU, rather than overall death. The review question is outlined in more detail in table 1 .

\section{METHODS}

We report our protocol using the Preferred Reporting Items for Systematic Reviews and Meta-Analyses Protocols (PRISMA-P) guidelines ${ }^{9}$ (online supplemental appendix 1-PRISMA-P checklist). Any amendments to the protocol will be made through PROSPERO. For this review, we will make use of the systematic review software DistillerSR (DistillerSR, Evidence Partners, Ottawa, Canada). For all processes from abstract and title screening to the final evidence synthesis, we will make use of the functionalities and built-in forms within DistillerSR.
If it is possible to combine the results of the included studies, we will also carry out a meta-analysis of the outcome of mortality in PICU.

\section{Search strategy}

The aim of the search is to identify any studies that have directly or indirectly addressed the association between sex and mortality in children admitted to intensive care.

The search strings were based on terms related to the population (children in intensive care), the exposure (sex) and the outcome (mortality).

The search terms were chosen to be sufficiently broad to capture all publications including those that focus on a particular subgroup of admissions to intensive care, for example admissions restricted to a particular primary diagnostic group only. Examples of the MeSH terms used were 'mortality or death', 'critical care or intensive care', 'sex or gender'. The terms were expanded where necessary to capture a wide range of studies. The search was not restricted to any languages, however, the full-text selection will exclude non-English articles. This is to provide an idea of the proportion of excluded non-English titles and abstracts that are deemed suitable for full text review. If there is a high proportion of such titles and abstracts, then an amendment to the strategy may be necessary to include articles published in non-English languages.

We carried out the search using the databases MEDLINE (via Ovid 1946-February week 4 2020), Embase (via Ovid 1980-2020week 52) and Web of Science (1985-2020) as these cover relevant clinical publications. The search strategy was refined after we reviewed the relevance of preliminary searches. The search strategies and the steps taken to refine the search along with the numbers of records found are detailed in figure 1 and online supplemental appendix 2.

Given the large number of initial records found, we followed the deduplication steps described by Shamseer $e t$ $a l^{10}$

\section{Study outcomes}

Primary outcome

- Mortality in PICU.

Secondary outcomes

- Length of Stay in PICU.

- Hospital mortality.

- Length of stay in hospital.

- Factors associated with differences in mortality rates for sexes.

Table 1 The review question following the PECO model

\begin{tabular}{ll}
\hline Population & Children aged $\mathbf{0}-\mathbf{1 7}$ years of age, and admitted to a paediatric intensive care unit \\
\hline Exposure & Sex is the exposure of interest \\
Comparison & Comparing male and female mortality rates \\
Outcome & Death within a paediatric intensive care unit \\
\hline
\end{tabular}

PECO, Population, Exposure, Comparison, Outcomes. 


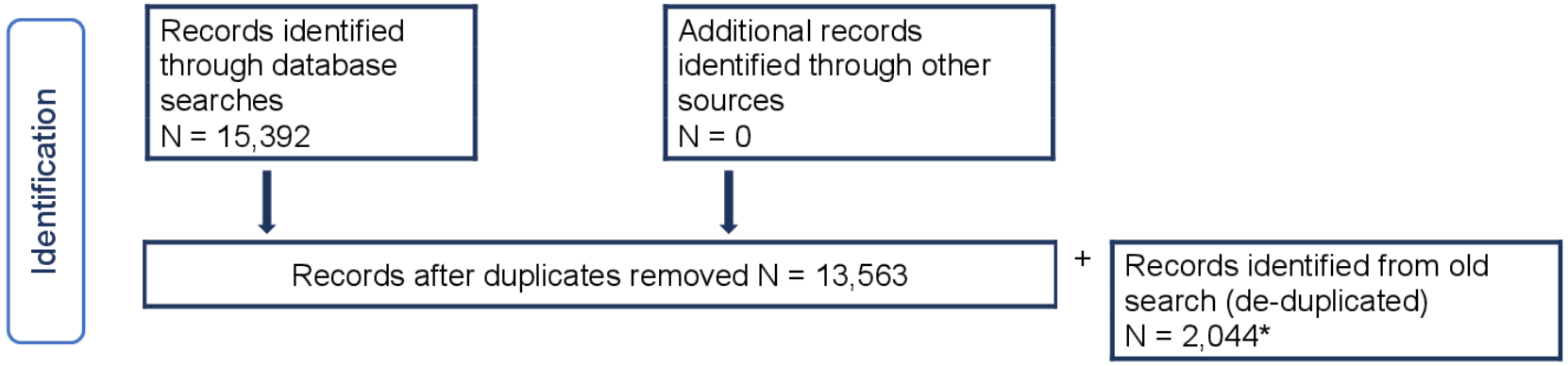

Merged sets of de-duplicated records N $=15,607$

Further de-duplication N = 14,219
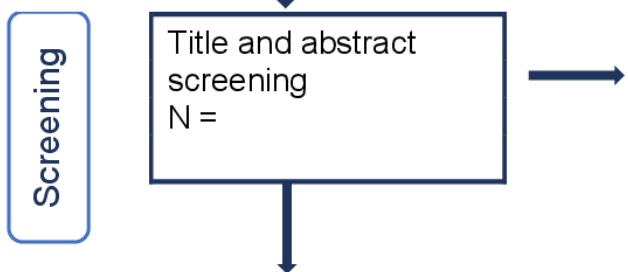

Records excluded $\mathrm{N}=$

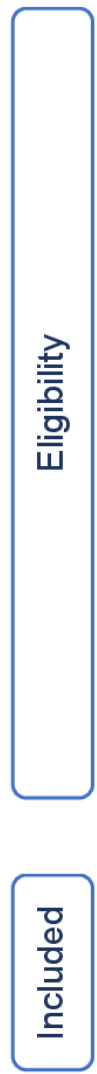

Stage 1 full-text articles assessed for eligibility

Full-text articles excluded, with reasons $\mathrm{N}=$

$N=$

----------

Full-text articles excluded, with reasons $\mathrm{N}=$

Studies remaining

after stage 1 full-text screening

$\mathrm{N}=$
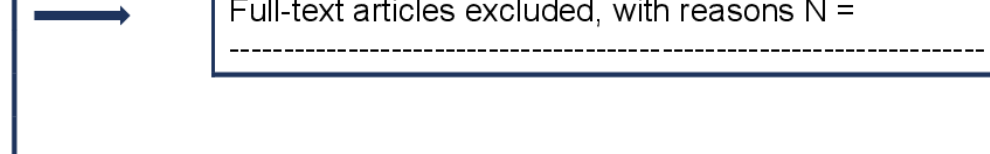

Full-text articles excluded, with reasons $N=$

\section{Studies remaining} after stage 2 full-text screening $\mathrm{N}=$

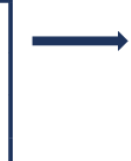

Full-text articles excluded, with reasons $N=$

Studies included in

evidence synthesis

and quantitative

synthesis (meta-

analysis)

$N=$

Additions to the original PRISMA Flow Diagram, Copyright @ 2020 , Evidence Partners Inc., All Rights Reserved.

Adapted from "Moher D, Liberati A, Tetzlaff J, Altman DG, The PRISMA Group (2009). Preferred Reporting Items for Systematic Reviews and MetaAnalyses: The PRISMA Statement. PLoS Med 6(7): e1000097. doi:10.1371/journal. pmed1000097"

For more information, visit: www.evidencepartners.com , www.prisma-statement.org

Figure 1 Data flow diagram. ${ }^{*}$ These records are detailed in online supplemental table A1 in online supplemental appendix 2.

PRISMA, Preferred Reporting Items for Systematic Reviews and Meta-Analyses. 


\section{Eligibility criteria}

Study inclusion criteria

We will include any observational study or reanalysis of randomised controlled trials reporting on the following:

- Children aged 0-17 years who have been admitted to a PICU/ICU.

- Reporting on mortality in PICU/ICU, either as a primary or secondary outcome.

- Reporting on sex differences (absolute or relative, as reported) in mortality, either as a main analysis or where sex is used as an adjustment variable for mortality.

\section{Study exclusion criteria}

- Studies meeting at least one of the exclusion criteria will be excluded.

- Studies exclusively addressing those admitted to neonatal units.

- Studies that only include premature or very low birthweight neonates.

- Studies that are only published in abstract form, or are review articles.

- Studies involving adult or mixed adult and paediatric populations. If the mixed adult and paediatric studies report separate results for the paediatric population, they will be considered.

- Studies that do not report PICU mortality as an outcome.

- Studies that do not report PICU mortality outcome by categories of sex.

- Studies not in English. However, the number of studies will be assessed after the title and abstract review stage. If more than $20 \%$ of the first stage full text reviews are not in English, a discussion between the reviewers will take place on whether the protocol needs to be updated to include these studies.

\section{Study screening mode}

Screening studies: applying inclusion and exclusion criteria

Before applying the study criteria, and to aid speeding the screening process, the lead author (OA) will remove irrelevant publication types such as conference abstracts, reviews and books. In the first instance, one reviewer will apply the inclusion and exclusion criteria to the title and abstract, to remove spurious citations. A sample of the reviewed titles and abstracts containing all the inclusions and a sample of the exclusions (twice the number of the inclusions) will be screened by the second reviewer. Discrepancies at the title and abstract review stage will be resolved by a third reviewer. The third reviewer will only screen the titles and abstracts with disagreements. We will review the full text of the articles selected from the title and abstract reviews in two stages. In the first stage, we will apply rapid exclusion of any articles that meet any of the exclusion criteria, see online supplemental appendix 3-stage 1 full-text screening tool. The first stage of full-text review will be applied by one reviewer and checked by a second. The remaining articles will go through a second stage full-text review and data extraction by one reviewer, then checked by a second reviewer.

\section{Screening studies: quality assurance process}

We will calculate a Kappa statistic for the agreement in inclusion/exclusions when applied to titles and abstracts, and to the first phase of full-text review level in order to measure agreement between the reviewers.

\section{Critical appraisal of included studies}

We will assess the reliability of included studies using the risk of bias in observational studies of exposures tool, ${ }^{11} 12$ which includes criteria for judging studies to be of high, unclear and low risk of bias. We will assess the studies for external validity using the same tool. Two reviewers will independently assess the methodological quality for eligible studies based on the criteria set out in table 2 . Any publication with a disagreement over the risk of bias between the two authors will be reviewed independently by the third reviewer.

\section{Critical appraisal mode}

Two viewers will apply the criteria of the critical appraisal independently and discrepancies will be resolved by a third reviewer.

\section{Data extraction strategy}

We will use the DistillerSR software for data extraction and make use of the built in data extraction templates.

Table 2 The study eligibility criteria following the PECO model

\begin{tabular}{|c|c|c|}
\hline PECO & Inclusion criteria & Exclusion criteria \\
\hline Exposure & $\begin{array}{l}\text { Sex used as a primary exposure for mortality } \\
\text { sex used as covariate for adjustment }\end{array}$ & $\begin{array}{l}\text { Mortality not reported by sex. } \\
\text { Sex as primary exposure or covariate for adjustment only in } \\
\text { non-mortality outcomes. }\end{array}$ \\
\hline Outcomes & $\begin{array}{l}\text { Primary: mortality in } \\
\text { PICU }\end{array}$ & Primary outcome not reported. \\
\hline
\end{tabular}

PECO, Population, Exposure, Comparison, Outcomes; PICU, paediatric intensive care unit. 
The proposed data extraction tool will be piloted by the three reviewers (OA, AF and LP) using two studies each before any amendments are made. Data extraction will be performed by the main reviewer, OA. Two reviewers (AF and LP) will share the task of second checking the extracted data.

We will tabulate the data items then generate a summary of studies' table. Data items of interest will be the age range of children admitted to critical care, if sex was used as a main exposure variable or one for adjustment, sample size of the study and the estimand used to compare mortality rates. The full data extraction sheet is available in online supplemental appendix 4-proposed data extraction tool and the risk of bias tool in online supplemental appendix 5-risk of bias Tool. Studies directly addressing the association will be eligible for data extraction. We will only report a summary of the estimates for studies where sex was used as an adjustment variable.

\section{Data analysis and synthesis}

We will carry out the analysis in Stata 16 or R software. We will carry out a narrative synthesis of the data, and depending on how mortality comparisons are calculated, the degree of similarities between the study designs, and the heterogeneity of the results, we may also summarise the estimates in a pooled effect. Although the outcome studied here is mortality, and therefore, quite clear to extract from the studies, the design and analysis of each study may vary considerably such that it would not be possible to combine the results in a meta-analysis.

We will consider a pooled effect if we have at least three studies with similar periods of follow-up and adjustment variables. We will consider analysis by subgroups if we have at least three studies with the same sub-population of primary diagnostic group, for example, studies conducted only on children with sepsis.

It may be possible to combine the crude estimate of studies with a similar length of stay. In which case we may combine the crude mortality estimates from individual studies in a meta-analysis to provide a pooled crude effect. If there are at least three studies, we will assess heterogeneity by calculating the Cochran's $Q$ statistic, and the $\mathrm{I}^{2}$ statistic to describe the percentage of variation across studies that is due to heterogeneity rather than chance.

We will extract the following data from each included study:

1. Publication date, country and setting (single centre or multi-centre) in which the study was conducted.

2. Study design and sample size.

3. Analysis of the entire PICU population or a subpopulation by primary diagnostic group.

4. Participant characteristics such as age range.

5. Features of the study: mortality (primary or secondary); type of effect measure (OR, HR or risk ratio (RR)); follow-up period. Secondary outcomes will also be extracted.

6. If sex was used as a primary exposure variable or as an adjustment variable
7. Reporting of estimates: adjusted, unadjusted and any adjustment variables used. The rationale for the inclusion of any adjustment variables.

8. Statistical model(s) used.

The effect size we report will depend on what the individual studies report. Possible estimands can be OR, RR, risk difference or HR.

We will extract the measure of association reported in each study along with its SE or CIs, depending on the subpopulation, the age group, the length of stay and the set of adjustment variables used in each study. We will present quantitative results from the included studies in tables and/or charts, and discuss them narratively.

\section{Sensitivity analyses}

We will carry out the following sensitivity analyses:

- Assess the impact of excluding studies with a high risk of bias.

- Assess the impact of excluding studies that involve only subpopulations of PICU.

- Should we exclude non-English publications, we will assess the impact of their exclusion.

\section{Dealing with missing data}

When the necessary data are not available in the included study or its incorporated studies, we will attempt to contact the authors of the primary studies.

\section{Ethics and dissemination}

Ethical approval is not required for this review as it will synthesise data from existing studies. This manuscript is a part of a larger data linkage study, for which Ethical approval was granted by the London - City \& East Research Ethics Committee, REC reference: 19/LO/1396, IRAS project ID: 214031. Dissemination will be via peerreviewed journals and via public and patient groups.

\section{Author affiliations}

${ }^{1}$ Department of Population, Policy and Practice, UCL GOS Institute of Child Health, London, UK

${ }^{2}$ Department of Paediatrics, Leeds Children's Hospital, Leeds, UK

${ }^{3}$ Department of Public Health and Infectious Diseases, University of Rome La Sapienza, Rome, Italy

${ }^{4}$ Department of Medical Statistics, LSHTM, London, UK

${ }^{5}$ Department of Global Health, Nagasaki University Institute of Tropical Medicine, Nagasaki, Japan

${ }^{6}$ Faculty of Medicine \& Health, University of Leeds, Leeds, UK

\section{Twitter Ofran Almossawi @ofran_a}

Acknowledgements We would like to thank members of the Project Advisory Group, Paul Saunders and Viki Ainsworth, for their contributions to the larger project and this manuscript in particular. We are grateful to our funder, the National Institute for Health Research, for their fellowship grant to Ofran Almossawi.

Contributors $O A$ conceived the idea for the literature review and drafted the protocol. BDS and RF reviewed and refined the protocol aims and objectives. $\mathrm{BDS}, \mathrm{RF}, \mathrm{AF}$ and $\mathrm{LP}$ reviewed, contributed to and approved the manuscript for the protocol. $\mathrm{OA}$ will conduct the literature search and $\mathrm{AF}$ and $\mathrm{LP}$ will review the search strategy and approve it. OA and AF will screen all the titles and abstracts. LP will resolve conflicts from the title and abstracts review. For the included full text publications, $\mathrm{OA}$ will extract the data and complete the risk of bias tool. AF and LP will check these steps. OA will conduct the analysis and draft the manuscript, LP will assist with the analysis. AF and LP will review and approve the final draft. 
Funding OA is funded by an NIHR Fellowship grant, ICA-CDRF-2018-04-ST2-049. This project is sponsored by the joint Research and Development (R\&D) at UCL Great Ormond Street Institute of Child Health.

\section{Competing interests None declared.}

Patient and public involvement statement This review is part of a larger research project with a Project Advisory Group (PAG). Members of the PAG have reviewed this manuscript, and will contribute to the final published review. Details of the main project can be found on the UCL Child Informatics Group Webpage.

Patient consent for publication Not required.

Provenance and peer review Not commissioned; externally peer reviewed.

Supplemental material This content has been supplied by the author(s). It has not been vetted by BMJ Publishing Group Limited (BMJ) and may not have been peer-reviewed. Any opinions or recommendations discussed are solely those of the author(s) and are not endorsed by BMJ. BMJ disclaims all liability and responsibility arising from any reliance placed on the content. Where the content includes any translated material, BMJ does not warrant the accuracy and reliability of the translations (including but not limited to local regulations, clinical guidelines, terminology, drug names and drug dosages), and is not responsible for any error and/or omissions arising from translation and adaptation or otherwise.

Open access This is an open access article distributed in accordance with the Creative Commons Attribution Non Commercial (CC BY-NC 4.0) license, which permits others to distribute, remix, adapt, build upon this work non-commercially, and license their derivative works on different terms, provided the original work is properly cited, appropriate credit is given, any changes made indicated, and the use is non-commercial. See: http://creativecommons.org/licenses/by-nc/4.0/.

ORCID iD

Ofran Almossawi http://orcid.org/0000-0001-6900-3590

\section{REFERENCES}

1 Royal College of Paediatrics and Child Health, CHR-UK Programme of Work at the MRC Centre of Epidemiology for Child Health, University College London Institute of Child Health. Child Health
Reviews UK - Overview of child deaths in the four UK countries 2013 https://www.rcpch.ac.uk/sites/default/files/CHR-UK_Retrospective_Epidemiological_Review_of_All-cause_Mortality_in_ CYP.pdf

2 United Nations, Department of Economic and Social Affairs, Population Division. Sex differentials in childhood mortality 2011 https://www.un.org/en/development/desa/population/publications/ mortality/sexdifferentials.asp

3 Paediatric Intensive Care Audit Network. PICANet 2019 annual report summary 2019 https://www.picanet.org.uk/wp-content/uploads/ sites/25/2019/12/PICANet-2019-Annual-Report-Summary_v1.0.pdf

4 Almossawi O, O'Brien ST, Parslow RC. Sex difference in infant mortality: a study of UK paediatric intensive care admissions over 11 years. Preprint (Version 1) available at Research Square 2020. doi:10.21203/rs.3.rs-86410/v1

5 Epstein D, Wong CF, Khemani RG, et al. Race/Ethnicity is not associated with mortality in the PICU. Pediatrics 2011;127:e588-97.

6 Johansson Frigyesi E, Andersson P, Frigyesi A. Boys have better short-term and long-term survival rates after intensive care admissions than girls. Acta Paediatr 2017;106:1973-8.

7 Fraser LK, Parslow R. Children with life-limiting conditions in paediatric intensive care units: a national cohort, data linkage study. Arch Dis Child 2018;103:540-7.

8 Almossawi O. Investigating differences in gender mortality for children admitted to UK critical care units. UCL Great Ormond Street Institute of Child Health, 2018. Available: https://www.ucl.ac.uk/ child-health/research/population-policy-and-practice-research-andteaching-department/cenb-clinical-10

9 Bramer WM, Giustini D, de Jonge GB, et al. De-duplication of database search results for systematic reviews in endnote. $J$ Med Libr Assoc 2016;104:240-3.

10 Shamseer L, Moher D, Clarke M, et al. Preferred reporting items for systematic review and meta-analysis protocols (PRISMA-P) 2015: elaboration and explanation. BMJ 2015;349:g7647.

11 Bero L, Chartres N, Diong J, et al. The risk of bias in observational studies of exposures (ROBINS-E) tool: concerns arising from application to observational studies of exposures. Syst Rev 2018;7:242.

12 Lansche J, Busse JW, Guyatt GH. Tool to assess risk of bias in cohort studies. Available: https://www.evidencepartners.com/ resources/methodological-resources/ 